\title{
Outbreak of gastroenteritis highlighting the diagnostic and epidemiological challenges of enteroinvasive Escherichia coli, County of Halland, Sweden, November 2017
}

Nina Lagerqvist ${ }^{1,2}$, Emma Löf $^{1,3}$, Theresa Enkirch ${ }^{1,2}$, Peter Nilsson $^{4}$, Adam Roth $^{1}$, Cecilia Jernberg $^{1}$

1. Public Health Agency of Sweden, Solna, Sweden

2. European Public Health Microbiology Training Programme (EUPHEM), European Centre for Disease Prevention and Control (ECDC), Stockholm, Sweden

3. European Programme for Intervention Epidemiology Training (EPIET), European Centre for Disease Prevention and Control $(E C D C)$, Stockholm, Sweden

4. Clinical Microbiology, County Hospital, Halmstad, Sweden

Correspondence: Cecilia Jernberg (cecilia.jernberg@folkhalsomyndigheten.se)

Citation style for this article:

Lagerqvist Nina, Löf Emma, Enkirch Theresa, Nilsson Peter, Roth Adam, Jernberg Cecilia . Outbreak of gastroenteritis highlighting the diagnostic and

epidemiological challenges of enteroinvasive Escherichia coli, County of Halland, Sweden, November 2017. Euro Surveill. 2020;25(9):pii=1900466. https://doi. org/10.2807/1560-7917.ES.2020.25.9.1900466

Article submitted on 16 Jul 2019 / accepted on 12 Dec 2019 / published on 05 March 2020

An outbreak of gastroenteritis with 83 cases occurred at a conference venue in November 2017 in Halland County, Sweden. Stool samples from two venue visitors and a symptomatic secondary case attributed to household transmission were PCR-positive for the ipaH gene, a target found in both Shigella spp. and enteroinvasive Escherichia coli (EIEC). EIEC was isolated from stool samples and whole genome sequencing analysis confirmed EIEC 096:H19 to be the aetiological agent. A cohort study was conducted among venue attendees and employees and the findings implicated contaminated leafy greens as the vehicle of infection, however, no microbiological evidence could support the study results. Here, we report the investigation into the first recorded EIEC outbreak in Sweden and illustrate the challenges associated with the differential laboratory diagnostics of Shigella/EIEC in an outbreak setting.

\section{Background}

Enteroinvasive Escherichia coli (EIEC) and Shigella spp. are both Gram-negative bacteria causing diarrheal disease worldwide $[1,2]$. The clinical presentations of these two pathogens are very similar $[3,4]$ and commonly manifested through diarrhoea, abdominal cramps, nausea and fever both in children and adults $[5,6]$. In addition to a similar clinical picture, EIEC and Shigella share laboratory features that can make it difficult to distinguish between them in routine clinical laboratory practice. Both pathogens are transmitted via the faecal-oral route and infections are frequently associated with consumption of contaminated food and water [7-10]. While Shigella is associated with large-scale food-borne outbreaks [11,12], outbreaks caused by EIEC are rarely recorded.

High prevalence of EIEC infections have been documented in rural areas and settings with poor sanitation in high-risk countries $[5,13]$ while EIEC infections in Europe are typically sporadic and travel related [14]. Nevertheless, a few EIEC outbreaks have been reported in Europe, with the most recent ones having occurred in Italy in 2012 [15] and in the United Kingdom (UK) in 2014 [16]. These outbreaks affected 109 cases and 157 probable cases, respectively, highlighting the fact that EIEC, like Shigella, has the capacity to cause large gastrointestinal disease outbreaks. The outbreak strain identified in these recent European outbreaks, EIEC 096: $\mathrm{H}_{19}$, is an emergent type of EIEC that has phenotypic characteristics more resembling those of noninvasive Escherichia coli ( $E$. coli) than those described for Shigella [17]. These characteristics are suggested to contribute to improved survival abilities as well as the ability to better adapt to different ecological niches [17].

Traditionally, culturing of faecal specimens has been the mainstay of laboratory diagnostics for enteric bacteria, and EIEC has been differentiated from Shigella by assessing a combination of several phenotypic characteristics, including biochemical, motility and serological traits $[18,19]$. This is now changing as PCRbased methods are becoming routine in many diagnostic laboratories [20]. In contrast to non-invasive E. coli, 
Onset of gastroenteritis symptoms of outbreak cases among conference and hotel venue attendees and personnel, County of Halland, Sweden, November $2017(\mathrm{n}=83)$

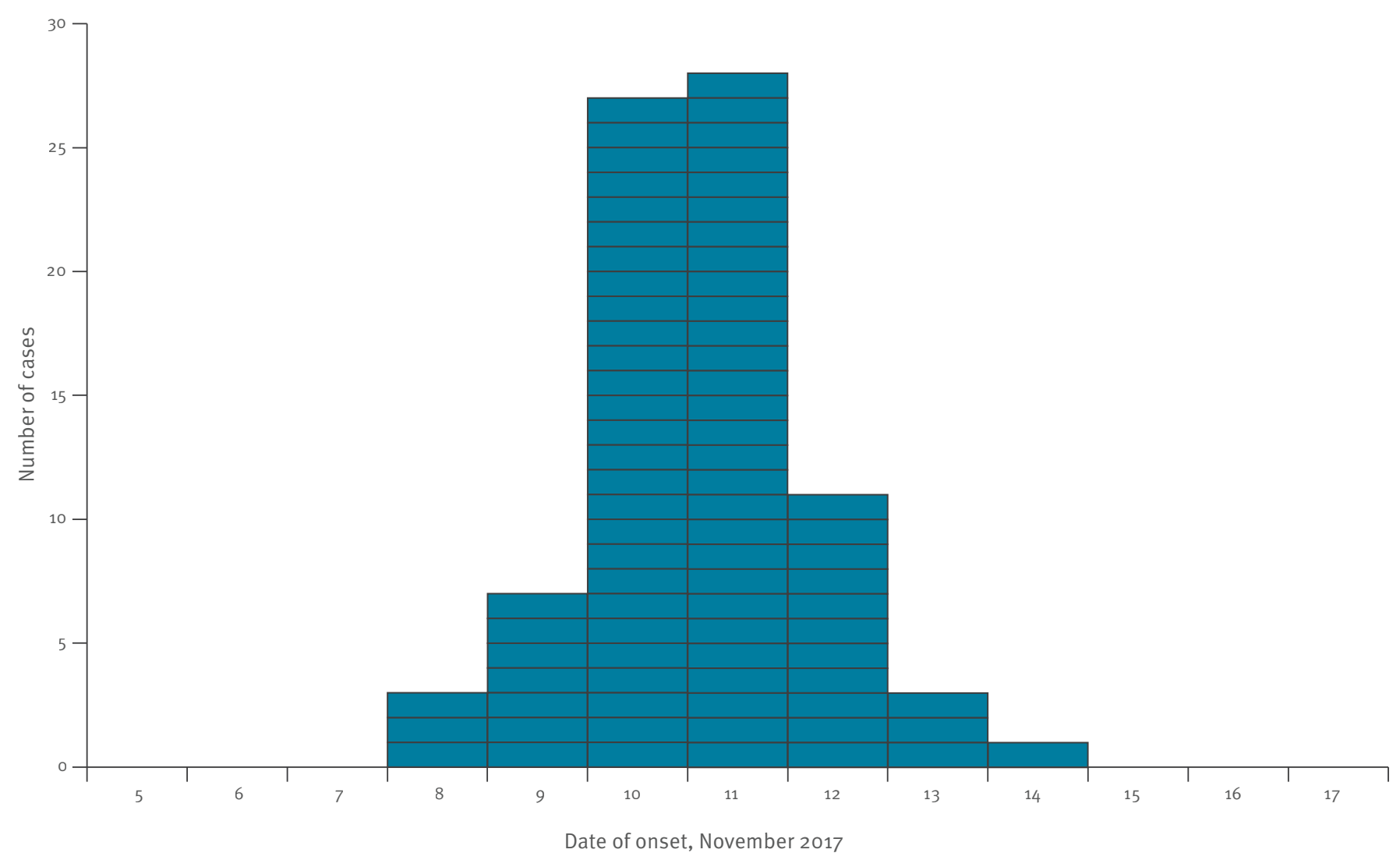

EIEC and Shigella can invade and multiply in intestinal epithelial cells [21], a process that is partially mediated by the products of the invasion plasmid antigen (ipa) genes [22]. For this reason, PCR targeting the ipaH gene can separate EIEC from other non-invasive $E$. coli, but cannot differentiate between EIEC and Shigella [23]. The lacY gene has been proposed as an additional molecular marker for which most $E$. coli are positive and Shigella is negative [24]. Its use as a PCR target in separating Shigella and EIEC is restricted to bacterial isolates since many faecal samples are lacY positive because of the presence of $E$. coli in the normal flora.

In Sweden, several clinical laboratories have shifted towards the use of direct PCR testing on faecal specimens as the primary diagnostic tool. However, most of these laboratories culture PCR-positive samples, so called PCR-guided culturing. Although culturing of PCR-positive faecal specimens is routinely performed, it can be difficult to obtain EIEC isolates since the morphology of EIEC strains on commonly used substrates can mimic the morphology of the enteric background flora, yellow colonies on xylose lysine deoxycholate (XLD) agar, rather than the morphology of Shigella, red colonies on XLD agar. Hence, separating EIEC from other bacteria in the normal flora usually requires additional laboratory procedures such as screening large numbers of colonies, which is considered too time consuming for most clinical laboratories. For this reason, it is likely that a patient with specimens that are $i p a H$ PCR-positive but culture negative would not be notified as a case if the diagnostic algorithm at the laboratory requires a detected Shigella isolate. In addition, PCR is a more sensitive method than culturing [25] and Shigella is known for its limited survival ability in faecal samples [26], which also may lead to samples being ipaH PCR-positive but culture negative.

Shigellosis is notifiable by law in Sweden as in the majority of countries in Europe [27]. In 2017, the incidence was 2.1 per 100,000 inhabitants in Sweden, and the majority of cases had been infected abroad [28]. The mandatory reporting of diseases allows the implementation of a series of public health actions, including public health management and surveillance activities, and helps define risk exposures. In contrast to shigellosis, reporting is not mandatory for EIEC and the occurrence of this pathogen in Sweden is currently unknown.

\section{Outbreak detection}

On 13 and 14 November 2017, local health authorities in Halland County and the Environmental Health Unit of Falkenberg Municipality received phone calls from three individuals who had developed gastrointestinal illness after visiting a conference and hotel venue on 8 
Attack rate and crude risk ratios for gastroenteritis among conference and hotel venue attendees and personnel, by meals, 8-10 November 2017, County of Halland, Sweden ( $n=398$ survey respondents)

\begin{tabular}{|c|c|c|c|c|c|c|c|c|c|c|}
\hline \multirow{2}{*}{ Date } & \multirow{2}{*}{ Exposure } & \multicolumn{3}{|c|}{ Exposed } & \multicolumn{3}{|c|}{ Non-exposed } & \multirow{2}{*}{ RR } & \multirow{2}{*}{$95 \% \mathrm{Cl}$} & \multirow{2}{*}{$\mathrm{p}$ value } \\
\hline & & Cases (n) & Total (N) & AR (\%) & Cases (n) & Total (N) & AR (\%) & & & \\
\hline \multirow{5}{*}{$8 \mathrm{Nov}$} & Breakfast & 11 & 63 & 18 & 72 & 335 & 22 & 0.81 & $0.46-1.44$ & 0.470 \\
\hline & Coffee break & 17 & 70 & 24 & 66 & 328 & 20 & 1.21 & $0.76-1.92$ & 0.436 \\
\hline & Lunch & 24 & 147 & 16 & 59 & 251 & 24 & 0.69 & $0.45-1.07$ & 0.089 \\
\hline & Afternoon coffee & 22 & 110 & 20 & 61 & 288 & 21 & 0.94 & $0.61-1.46$ & 0.795 \\
\hline & Dinner & 26 & 103 & 25 & 57 & 295 & 19 & 1.31 & $0.87-1.96$ & 0.203 \\
\hline \multirow{5}{*}{9 Nov } & Breakfast & 32 & 127 & 25 & 51 & 271 & 19 & 1.34 & $0.91-1.97$ & 0.144 \\
\hline & Coffee break & 65 & 244 & 27 & 18 & 154 & 12 & 2.28 & $1.41-3.69$ & 0.000 \\
\hline & Lunch & 75 & 301 & 25 & 8 & 97 & 8 & 3.02 & $1.51-6.04$ & 0.000 \\
\hline & Afternoon coffee & 54 & 192 & 28 & 29 & 206 & 14 & 2.00 & $1.33-3.00$ & 0.001 \\
\hline & Dinner & 27 & 93 & 29 & 56 & 305 & 18 & 1.58 & $1.06-2.35$ & 0.027 \\
\hline \multirow{5}{*}{$10 \mathrm{Nov}$} & Breakfast & 27 & 80 & 34 & 56 & 318 & 18 & 1.92 & $1.30-2.83$ & 0.001 \\
\hline & Coffee break & 24 & 68 & 35 & 59 & 330 & 18 & 1.97 & $1.33-2.93$ & 0.001 \\
\hline & Lunch & 31 & 98 & 32 & 52 & 300 & 17 & 1.82 & $1.25-2.67$ & 0.002 \\
\hline & Afternoon coffee & 8 & 25 & 36 & 74 & 373 & 20 & 1.81 & $1.04-3.18$ & 0.054 \\
\hline & Dinner & 6 & 23 & 26 & 77 & 375 & 21 & 1.27 & $0.62-2.60$ & 0.525 \\
\hline
\end{tabular}

AR: attack rate; $\mathrm{Cl}$ : confidence interval; RR: risk ratio.

to 10 November. The Public Health Agency of Sweden (PHAS) was notified about the suspected food-borne outbreak characterised by acute diarrhoea. Additional phone interviews with 44 individuals who had attended the venue revealed that 22 persons had experienced gastrointestinal symptoms after visiting the venue for 1 day or more during the period 8 to 10 November. An outbreak investigation team was convened with representatives from the local health authorities, PHAS, the Swedish National Food Agency and the Environmental Health Unit. The aim was to investigate the magnitude, identify the causative agent and localise the source of the outbreak.

Here, we report the findings from the outbreak investigation and illustrate the challenges associated with the differential diagnostics of EIEC and Shigella.

\section{Methods}

\section{Epidemiological investigation}

We conducted a cohort study to investigate the outbreak, and an online questionnaire was created to collect data on the dates of venue attendance, date of symptom onset, symptoms and food consumed. The food items specified in the questionnaire were obtained from the restaurant's menu. Email addresses of venue visitors and employees were collected via contact persons for the visiting parties or the venue's proprietors, respectively. Information on age was not collected in the questionnaire. The contacted parties visited the venue in connection to work. A link to a web-based questionnaire was sent on 17 and 20 November to 554 email addresses, and a reminder was distributed on 23 November.

\section{Case definition}

A case was defined as an individual who consumed food and/or beverage at the conference venue during the period 8 to 10 November 2017, and reported symptoms of gastrointestinal illness including abdominal pain, nausea, diarrhoea (more than three loose stools in 24 hours), bloody diarrhoea and/or vomiting within 7 days after attending the venue, i.e. symptom onset before 15,16 or 17 November.

\section{Statistical analysis}

Univariate analysis of exposures was performed using the CSTABLE command [29] in Stata version 14.0 (Stata Corporation, College Station, Texas, United States (US)). We obtained attack rates (AR), risk ratios (RR) with $95 \%$ confidence intervals $(95 \% \mathrm{Cl})$ and $\mathrm{p}$ values (p). $P$ values below 0.05 were considered significant. Differences of rates among the exposed and unexposed were analysed using chi-squared test.

\section{Microbiological investigations}

The local clinical microbiological laboratory received stool samples from two outbreak cases and a suspected secondary case, and performed routine screening methods for gastrointestinal pathogens. An in-house real-time PCR approach was used targeting genes from a variety of gastrointestinal pathogens, including the ipaH gene. Guided by the PCR finding of the ipaH target, attempts were made to isolate Shigella from two outbreak samples by inoculation on Acumedia XLD agar (Neogen, Lansing, 
Attack rate and crude risk ratios for gastroenteritis among conference and hotel venue attendees and personnel, by food item served at lunch, 9 November 2017, County of Halland, Sweden ( $\mathrm{n}=301$ survey respondents)

\begin{tabular}{|c|c|c|c|c|c|c|c|c|c|}
\hline \multirow{2}{*}{ Exposure } & \multicolumn{3}{|c|}{ Exposed } & \multicolumn{3}{|c|}{ Unexposed } & \multirow{2}{*}{$\mathrm{RR}$} & \multirow{2}{*}{$95 \% \mathrm{Cl}$} & \multirow{2}{*}{$p$ value } \\
\hline & Cases (n) & Total (N) & AR (\%) & Cases (n) & Total (N) & AR (\%) & & & \\
\hline Root vegetables (salad buffet) & 50 & 152 & 33 & 25 & 149 & 17 & 1.96 & $1.28-2.99$ & 0.001 \\
\hline Beans (salad buffet) & 30 & 78 & 38 & 45 & 223 & 20 & 1.91 & $1.30-2.80$ & 0.001 \\
\hline Plaice & 51 & 160 & 32 & 24 & 141 & 17 & 1.87 & $1.22-2.88$ & 0.003 \\
\hline Julienne vegetables ${ }^{a}$ & 29 & 81 & 36 & 28 & 140 & 20 & 1.79 & $1.15-2.78$ & 0.010 \\
\hline Raw vegetables (salad buffet) & 26 & 70 & 37 & 49 & 231 & 21 & 1.75 & $1.18-2.59$ & 0.007 \\
\hline $\begin{array}{l}\text { Tomato and onion salad (salad } \\
\text { buffet) }\end{array}$ & 48 & 152 & 32 & 27 & 149 & 18 & 1.74 & $1.15-2.64$ & 0.007 \\
\hline Bean salad & 21 & 55 & 38 & 54 & 246 & 22 & 1.74 & $1.15-2.62$ & 0.012 \\
\hline Leafy greens (salad buffet) & 45 & 152 & 30 & 30 & 149 & 20 & 1.47 & $0.98-2.20$ & 0.058 \\
\hline
\end{tabular}

AR: attack rate; $\mathrm{Cl}$ : confidence interval; $\mathrm{RR}$ : risk ratio.

${ }^{a} n=221$

Michigan, US). Only yellow colonies grew on the XLD agar, indicating that no Shigella could grow from the specimens. Additional culturing attempts directed towards Shigella and EIEC were made, including dilution of samples for plate inoculation and increasing the number of colonies per sample picked and screened by PCR targeting the ipaH gene. Three ipaH PCR-positive isolates were sent to PHAS for species determination and serotyping. The isolates were phenotypically analysed using a biochemical panel of six different parameters and serotyped by agglutination using pooled antisera, all according to reference methodology [30].

\section{Whole genome sequencing}

Bacterial DNA was extracted using the MagDEA Dx SV reagent kit and the magLEAD instrument (Precision System Science, Chiba, Japan) and sequenced on the Ion Torrent $\mathrm{S}_{5} \mathrm{XL}$ platform (Thermo Fisher Scientific, Waltham, Massachusetts, US). Library and template preparations were performed according to the manufacturer's instructions (Thermo Fisher Scientific). The raw data was assembled to contigs using CLC assembly cell version 4.4.2 (Qiagen Bioinformatics, Hilden, Germany). Raw reads were then mapped to the assembly and the average coverage was calculated. Genomes with incorrect total size $(+/-20 \%)$ or low coverage ( $20 x)$ were flagged as failed quality control. Multilocus sequence typing (MLST) was calculated by mapping the raw reads to a reference sequence for each loci of the Warwick $E$. coli 7-gene MLST scheme. Consensus sequences were called and compared with the allele reference databases using BLAST+version 2.2.30. The molecular serotype was determined by comparing contigs to the SerotypeFinder version 1.0 database (Technical University of Denmark (DTU), Lyngby, Denmark) using BLAST+version 2.2.30, the wzx and wzy genes for O-type and the fliC gene for H-type. Single nt polymorphisms (SNPs) were called based on an assembly of one of the outbreak strains using CLC assembly cell (minimum 10x coverage, $90 \%$ read consensus). Minimum spanning trees were generated using MSTgold [31] and recombinations were filtered by looking for SNPs with a pairwise distance of $500 \mathrm{nt}$. The sequences were deposited in the European Nt Archive (ENA) under accession numbers ERS3907567, ERS3907568 and ERS3907569.

\section{Environmental investigations}

On 15 November, environmental health officers inspected the restaurant at the venue and reviewed its routines for food handling, storage and kitchen hygiene, as well as collected leftover food samples that had not been consumed or thrown away. Sampled food items were cooked duck, mousse, blueberry meringue, vanilla pastry, pickled red cabbage, bleak roe and freeze-dried blueberries. The food samples were tested for E. coli at commercial laboratories. Leftover frozen dill was later collected and tested for $E$. coli at the Swedish National Food Agency.

\section{Ethical statement}

Written consent was obtained from all participants who provided questionnaire answers. Ethical approval was not required as the investigation was performed under a mandate of The Public Health Agency of Sweden in its remit to undertake outbreak investigations regarding national communicable disease control in the interest of public health.

\section{Results}

\section{Descriptive analysis}

From the list of 554 eligible individuals who received the web-based questionnaire, 351 visitors and 47 staff members completed the survey (response rate: $72 \%$ ). Of these, $57 \%(n=228)$ were female and three did not provide information on sex. The case definition was met by 83 individuals ( 78 visitors and 5 staff members), of whom $67 \%(n=56)$ were female and one did not provide information on sex. The overall attack rate was $21 \%(83 / 398)$, and was higher for women $(25 \%$; $56 / 228)$ than men $(16 \% ; 26 / 167)$. The date of onset 
of symptoms ranged from 8 to 14 November, with the peak occurring on 10 and 11 November (Figure). Three cases did not provide information on symptom onset. Fourteen cases reported their symptoms to last for 1 day or less, 11 cases each reported symptom duration of 2 or 3 days, five cases reported 4 days, but a majority of the cases $(n=42 ; 51 \%)$ were symptomatic for 5 or more days. Symptoms reported by the cases were abdominal pain $(n=74 ; 89 \%)$, bloody diarrhoea $(n=4$; $4.8 \%)$, diarrhoea $(n=73 ; 88 \%)$, nausea $(n=55 ; 66 \%)$ and vomiting $(n=8 ; 9.6 \%)$.

\section{Food exposure}

The venue served breakfast, lunch, dinner and snacks during coffee breaks to hotel and conference attendants and staff members, and was open to the public for lunch and dinner servings. An estimated 1,000 portions were served between 8 and 10 November. Of the 398 respondents, 173 individuals including 32 cases attended the venue on 8 November (RR: 0.82 ; $95 \%$ $\mathrm{Cl}: 0.55-1.21 ; \mathrm{p}=0.310), 341$ individuals including 80 cases attended the venue on 9 November (RR:4.46; $95 \% \mathrm{Cl}: 1.46-13.63 ; \mathrm{p}=0.002)$, and 129 individuals including 35 cases attended the venue 10 November (RR: $1.52 ; 95 \% \mathrm{Cl}: 1.04-2.23 ; \mathrm{p}=0.033$ ). None of the 41 individuals who solely visited the venue on 8 November experienced gastrointestinal symptoms and exposure to meals served on 8 November were not significantly associated with illness (Table 1). The highest risk ratios were observed for attending meals served between the morning and afternoon coffee breaks on 9 November, and food consumption at lunch (Table 1). Food items served on 9 and 10 November were analysed for association with illness. Food items significantly associated with disease were served during lunch on 9 November and are shown in Table 2. No strong association was observed between illness and consumption of a specific food item (Table 2). The ambiguous results led to additional interviews with kitchen staff, who informed the outbreak investigation team that a variety of leafy green products, including baby spinach, rocket salad and mixed salads, were used as garnish on or in dishes (previously undisclosed ingredients).

\section{Microbiological investigations}

Stool samples from three individuals, two venue visitor cases and a secondary case attributed to household transmission, tested positive for the ipaH-gene and were PCR-negative for other common gastrointestinal pathogens. Isolates were successfully retrieved from these three stool samples by culturing at the local laboratory and were sent to the reference laboratory for further characterisation. The outbreak strain was positive for $\beta$-galactosidase and lysine decarboxylase activity (LDC), fermented sugars such as glucose and mannose, was motile, and tested negative in Shigella agglutination tests. For this reason, the isolates were defined as EIEC.

Data obtained from whole genome sequencing (WGS) was used to determine the molecular serotype, the sequence type (ST) and to assess genetic similarity among the isolates. The isolates were $E$. coli serotype 096:H19, a known EIEC serotype, and were classified as ST99. The three isolates showed high genomic sequence similarity (0-1 SNP difference, $99.8 \%$ of the genome). Isolates collected from the two venue visitors showed indistinguishable genomes (no SNP difference) while the genome of the isolate collected from the secondary case differed from the other two in one SNP. We compared the genomic sequences obtained during this outbreak to published sequences [17] and the SNP-based phylogeny showed large genomic differences (>100 SNPs) between this outbreak strain and previously published EIEC 096:H19 outbreak strains (data not shown).

None of the food items sent for testing at the commercial laboratories or the frozen dill tested at the Swedish National Food Agency tested positive for E.coli.

\section{Outbreak control measures}

Representatives from the Environmental Health Unit performed a post-outbreak inspection of the kitchen and had no remarks. Officers performing routine inspections earlier that year came to the same conclusion. Perishable food items served between 8 and 10 November were already consumed or had been discarded when the outbreak control team arrived. Food inspectors traced back suspected food items including baby spinach, rocket salad and mixed leafy greens (including rocket salad, baby spinach and red leafy greens). Trace-back investigations identified a distributor who delivered these items to the venue on 7 November (baby spinach and mixed leafy greens), 8 November (rocket salad and mixed leafy greens) and 9 November (baby spinach, rocket salad and mixed leafy greens). The food items were received from a producer who trades with leafy greens and packages pre-washed, ready-to-eat leafy greens in bags, with products delivered 7 to 9 November originating from Italy and Sweden. The producer reported that they did not have any indication from their own microbiological testing of contaminated leafy greens during the reported dates and they had not received any complaints from other buyers. For these reasons, further control measures were not undertaken.

\section{Discussion}

This outbreak, with 83 self-reported cases and one secondary case attributed to household transmission, is the first reported EIEC outbreak in Sweden. The result of the outbreak investigation was inconclusive and no food item could be directly linked to the outbreak. However, leafy greens were suspected to be the vehicle of infection as this food item was present in a number of dishes associated with disease. The attack rate $(21 \%)$ was high, also suggesting that the causative agent may have been present in many of the dishes. There was no microbiological evidence to identify the source or vehicle of infection, however, consumption of contaminated salad greens have been associated with 
previous EIEC outbreaks $[15,16]$. The trace-back of the leafy greens did not implicate that these products were contaminated before arriving at the venue. However, this scenario cannot be excluded since several reports have shown that many outbreaks involving fresh produce are linked to contamination at the field level [32].

EIEC outbreaks have shown to affect individuals of all ages $[15,16]$. A weakness of our study is that we did not collect information on age in the online questionnaire. However, as we only received contact information to venue employees or individuals visiting the venue in connection to work, all individuals were of working age. In an outbreak reported in the UK in 2014, most of the food handlers who tested positive for EIEC were asymptomatic carriers and investigators identified inadequate handwashing facilities and food handling practices at the restaurant [16]. In the current outbreak, all five staff members that met the case definition had symptom onset at least 1 day after the first reported case among venue visitors. A limitation to our study is that we did not perform any interviews with kitchen staff regarding previous disease and travel history, and no members of staff were tested for asymptomatic EIEC carriage. The reason for this was that the outbreak ended quickly, suggesting that the source had been removed, and the officers inspecting the kitchen had no remarks on the facilities or the practices employed by the kitchen staff.

Despite the prompt notification to the reference laboratory and the efforts taken by the local clinical laboratory to retrieve isolates, i.e. implementing procedures to isolate EIEC to support the outbreak investigation, and obtaining the results from the WGS analysis, the definite result from the microbiological investigation was not available until 2 to 3 weeks after the outbreak was resolved. In Sweden, few laboratories actively search for EIEC in samples PCR-positive for ipaH and the probability of isolating EIEC during attempts to isolate Shigella is low. Further, culturing of Shigella may fail because of the pathogen's limited survival ability in faecal samples [26]. For this reason, a number of ipaH-positive samples will likely be culture-negative even if routines to isolate EIEC are implemented. As culture-independent diagnostics, i.e. PCR diagnostic testing, is becoming the method of choice at primary clinical laboratories, members of an outbreak investigation team need to be aware of the limitations of an ipaH PCR-positive finding and that a definite microbiological confirmation can take time, or will never be achieved.

This outbreak was identified because of the high number of persons falling ill during a short time period and because they all could quickly be linked to the same venue. Only two cases and one household contact were laboratory-confirmed. This can possibly be explained by several factors, including mild cases not seeking medical care, samples not being taken in patients presenting with short-term gastrointestinal symptoms or cases not being notified in the official reporting system because samples were only ipaH positive.

Despite the limited number of laboratory confirmed cases, we concluded EIEC to be the disease-causing pathogen. This is based on the absence of other common gastrointestinal pathogens in the collected stool specimens and the results of the WGS analysis revealing almost identical genomic sequences of the three EIEC isolates. WGS is becoming the routine analysis tool for typing at many public health reference laboratories [33]. The continuously increased use of WGS does not only allow identification of clustering among outbreak cases as shown in our study, but also provides knowledge on the genetic distribution of EIEC. This improves our understanding of what specific serotypes and additional marker genes are associated with EIEC, possibly enabling better molecular differentiation of Shigella and EIEC in the future.

In Sweden, the occurrence of EIEC is currently unknown and no information on the distribution of the specific outbreak strain was available to the outbreak investigation team, i.e. it is unknown if EIEC 096: $\mathrm{H}_{19}$ is circulating in Sweden or if this strain was introduced via an imported food item. This specific EIEC serotype of ST99 was first reported as the disease-causing pathogen in an outbreak in Italy in 2012, and has since been implicated in two outbreaks in the UK and a sporadic travel-related case in Spain [17]. The 096: $\mathrm{H}_{19}$ serotype of ST99 is considered a new emerging virulent EIEC strain [19] and differs from other traditional EIEC and Shigella strains in many phenotypic tests as it is more reactive, e.g. ferments glucose, is positive in LDC and is motile [17]. This was also shown in the present investigation. Emerging EIEC strains such as EIEC 096:H19, which phenotypically resembles $E$. coli more than Shigella which could enable improved survival abilities [17], could potentially contribute to an increase in food-borne outbreaks caused by EIEC in the future. This necessitates improved laboratory preparedness and consensus on recommendations for public health measures of PCR-positive Shigella/EIEC faecal samples.

\section{Acknowledgements}

We gratefully acknowledge I Henderström, K Schönmeyr and A Rasmusson for excellent technical assistance. We also thank A Gustafsson, S Hautakangas, M Vang and K Alness at the Environmental Health Unit in Falkenberg and C Flink at the National Food Agency for excellent technical assistance and valuable scientific advice.

Conflict of interest

None declared. 


\section{Authors' contributions}

Conceived and designed the study: NL, CJ, EL. Analysed the data: NL, EL. Performed laboratory work: PN. Wrote the paper: NL, CJ, EL, TE, PN, AR.

\section{References}

1. Croxen MA, Law RJ, Scholz R, Keeney KM, Wlodarska M, Finlay $B B$. Recent advances in understanding enteric pathogenic Escherichia coli. Clin Microbiol Rev. 2013;26(4):822-80. https://doi.org/10.1128/CMR.00022-13 PMID: 24092857

2. Baker S, The HC. Recent insights into Shigella. Curr Opin Infect Dis. 2018;31(5):449-54. https://doi.org/10.1097/ QC0.0000000000000475 PMID: 30048255

3. DuPont HL, Formal SB, Hornick RB, Snyder MJ, Libonati JP, Sheahan DG, et al. Pathogenesis of Escherichia coli diarrhea. N Engl J Med. 1971;285(1):1-9. https://doi.org/10.1056/ NEJM197107012850101 PMID: 4996788

4. Taylor DN, Echeverria P, Pál T, Sethabutr O, Saiborisuth S, Sricharmorn S, et al. The role of Shigella spp., enteroinvasive Escherichia coli, and other enteropathogens as causes of childhood dysentery in Thailand. J Infect Dis. 1986;153(6):1132 8. https://doi.org/10.1093/infdis/153.6.1132 PMID: 3517189

5. Vieira N, Bates SJ, Solberg OD, Ponce K, Howsmon R, Cevallos W, et al. High prevalence of enteroinvasive Escherichia coli isolated in a remote region of northern coastal Ecuador. Am J Trop Med Hyg. 2007;76(3):528-33. https://doi.org/10.4269/ ajtmh.2007.76.528 PMID: 17360879

6. Agbonlahor DE, Odugbemi TO. Enteropathogenic, enterotoxigenic and enteroinvasive Escherichia coli isolated from acute gastroenteritis patients in Lagos, Nigeria. Trans R Soc Trop Med Hyg. 1982;76(2):265-7. https://doi. org/10.1016/0035-9203(82)90293-0 PMID: 7048653

7. Gordillo ME, Reeve GR, Pappas J, Mathewson JJ, DuPont $\mathrm{HL}$, Murray BE. Molecular characterization of strains of enteroinvasive Escherichia coli $\mathrm{O}_{143}$, including isolates from a large outbreak in Houston, Texas. J Clin Microbiol. 1992;30(4):889-93. https://doi.org/10.1128/JCM.30.4.889893.1992 PMID: 1349307

8. Marier R, Wells J, Swanson R, Callahan W, Mehlman I. An outbreak of enteropathogenic Escherichia coli foodborne disease traced to imported French cheese. Lancet. 1973;302(7842):1376-8. https://doi.org/10.1016/ S0140-6736(73)93335-7

9. Snyder JD, Wells JG, Yashuk J, Puhr N, Blake PA. Outbreak of invasive Escherichia coli gastroenteritis on a cruise ship. Am J Trop Med Hyg. 1984;33(2):281-4. https://doi.org/10.4269/ ajtmh.1984.33.281 PMID: 6370005

10. Kotloff KL, Riddle MS, Platts-Mills JA, Pavlinac P, Zaidi AKM. Shigellosis. Lancet. 2018;391(10122):801-12. https://doi. org/10.1016/S0140-6736(17)33296-8 PMID: 29254859

11. Rooney RM, Cramer EH, Mantha S, Nichols G, Bartram JK, Farber JM, et al. A review of outbreaks of foodborne disease associated with passenger ships: evidence for risk management. Public Health Rep. 2004;119(4):427-34. https:// doi.org/10.1016/j.phr.2004.05.007 PMID: 15219800

12. Nygren BL, Schilling KA, Blanton EM, Silk BJ, Cole DJ, Mintz ED. Foodborne outbreaks of shigellosis in the USA, 1998-2008. Epidemiol Infect. 2013;141(2):233-41. https://doi.org/10.1017/ So950268812000222 PMID: 22361246

13. Taylor DN, Echeverria P, Sethabutr O, Pitarangsi C, Leksomboon U, Blacklow NR, et al. Clinical and microbiologic features of Shigella and enteroinvasive Escherichia coli infections detected by DNA hybridization. J Clin Microbiol. 1988;26(7):1362-6. https://doi.org/10.1128/JCM.26.7.13621366.1988 PMID: 3045152

14. Niyogi SK. Shigellosis. J Microbiol. 2005;43(2):133-43. PMID: 15880088

15. Escher M, Scavia G, Morabito S, Tozzoli R, Maugliani A, Canton $\mathrm{S}$, et al. A severe foodborne outbreak of diarrhoea linked to a canteen in Italy caused by enteroinvasive Escherichia coli, an uncommon agent. Epidemiol Infect. 2014;142(12):2559-66. https://doi.org/10.1017/So950268814000181 PMID: 24534429

16. Newitt S, MacGregor V, Robbins V, Bayliss L, Chattaway MA, Dallman T, et al. Two Linked Enteroinvasive Escherichia coli Outbreaks, Nottingham, UK, June 2014. Emerg Infect Dis. 2016;22(7):1178-84. https://doi.org/10.3201/eid2207.152080 PMID: 27314432

17. Michelacci V, Prosseda G, Maugliani A, Tozzoli R, Sanchez $S$, Herrera-León S, et al. Characterization of an emergent clone of enteroinvasive Escherichia coli circulating in Europe. Clin Microbiol Infect. 2016;22(3):287.e11-9. https://doi. org/10.1016/j.cmi.2015.10.025 PMID: 26551840
18. van den Beld MJ, Reubsaet FA. Differentiation between Shigella, enteroinvasive Escherichia coli (EIEC) and noninvasive Escherichia coli. Eur J Clin Microbiol Infect Dis. 2012;31(6):899-904. https://doi.org/10.1007/s10096-011-13957 PMID: 21901636

19. Pasqua M, Michelacci V, Di Martino ML, Tozzoli R, Grossi $M$, Colonna B, et al. The intriguing evolutionary journey of enteroinvasive E. coli (EIEC) toward pathogenicity. Front Microbiol. 2017;8:2390. https://doi.org/10.3389/ fmicb.2017.02390 PMID: 29259590

20. Shea S, Kubota KA, Maguire H, Gladbach S, Woron A, Atkinson-Dunn R, et al. Clinical Microbiology Laboratories' Adoption of Culture-Independent Diagnostic Tests Is a Threat to Foodborne-Disease Surveillance in the United States. J Clin Microbiol. 2016;55(1):10-9. https://doi.org/10.1128/JCM.0162416 PMID: 27795338

21. Hornick RB, DuPont HL, Music SI, Snyder MJ, Libonati JP. Investigations into the pathogenesis of diarrheal diseases. Trans Am Clin Climatol Assoc. 1971;82:141-7. PMID: 4934015

22. Hartman AB, Venkatesan M, Oaks EV, Buysse JM. Sequence and molecular characterization of a multicopy invasion plasmid antigen gene, ipaH, of Shigella flexneri. I Bacteriol. 1990;172(4):1905-15. https://doi.org/10.1128/JB.172.4.19051915.1990 PMID: 1690703

23. Sethabutr O, Venkatesan M, Murphy GS, Eampokalap B, Hoge CW, Echeverria P. Detection of Shigellae and enteroinvasive Escherichia coli by amplification of the invasion plasmid antigen H DNA sequence in patients with dysentery. J Infect Dis. 1993;167(2):458-61. https://doi.org/10.1093/ infdis/167.2.458 PMID: 8421181

24. Løbersli I, Wester AL, Kristiansen Å, Brandal LT. Molecular Differentiation of Shigella Spp. from Enteroinvasive E. Coli. Eur J Microbiol Immunol (Bp). 2016;6(3):197-205. https://doi. org/10.1556/1886.2016.00004 PMID: 27766168

25. von Seidlein L, Kim DR, Ali M, Lee H, Wang X, Thiem VD, et al. A multicentre study of Shigella diarrhoea in six Asian countries: disease burden, clinical manifestations, and microbiology. PLoS Med. 2006;3(9):e353. https://doi.org/10.1371/journal. pmed.0030353 PMID: 16968124

26. Watkins LKF, Appiah GD. Chapter 3: Shigellosis. In: Yellow book. Health Information for International Travel. Atlanta: Centers for Disease Control and Prevention [Accessed: 28 Feb 2019]. Available from: https://wwwnc.cdc.gov/travel/ yellowbook/2018/infectious-diseases-related-to-travel/ shigellosis

27. European Centre for Disease Prevention and Control (ECDC). Shigellosis. In: ECDC. Annual epidemiological report for 2016 Stockholm: ECDC; 2018. Available from: https://www.ecdc. europa.eu/sites/default/files/documents/AER for_2016shigellosis.pdf

28. Folkhälsomyndigheten. Anmälningspliktiga smittsamma sjukdomar i Sverige 2017 - epidemiologisk årsrapport, tabellsamling. [Notifiable communicable diseases in Sweden 2017- Epidemiological report]. Solna: Folkhälsomyndigheten; 2018. [Accessed: 29 Nov 2018]. Swedish. Available from: https://www.folkhalsomyndigheten. se/publicerat-material/publikationsarkiv/a/ anmalningspliktiga-sjukdomar-sverige-2017/

29. Desve G, Makary P. CSTABLE: Stata module to calculate summary table for cohort study. Statistical Software Components S456879. Boston: Boston College Department of Economics; 2007. Available from: https://ideas.repec.org/c/ boc/bocode/s456879.html

30. Folkhälsomyndigheten. Referensmetodik för laboratoriediagnostik vid kliniskt mikrobiologiska laboratorier. [Reference methodology for laboratory diagnostics in clinical microbiological laboratories]. Solna: Folkhälsomyndigheten. [Accessed: 30 Nov 2018]. Swedish. Available from: http://referensmetodik.folkhalsomyndigheten.se/w/ Shigella-laboratoriediagnostik

31. Salipante SJ, Hall BG. Inadequacies of minimum spanning trees in molecular epidemiology. J Clin Microbiol. 2011;49(10):356875. https://doi.org/10.1128/JCM.00919-11 PMID: 21849692

32. Lynch MF, Tauxe RV, Hedberg CW. The growing burden of foodborne outbreaks due to contaminated fresh produce: risks and opportunities. Epidemiol Infect. 2009;137(3):307-15 https://doi.org/10.1017/So950268808001969 PMID: 19200406

33. Revez J, Espinosa L, Albiger B, Leitmeyer KC, Struelens MJECDC National Microbiology Focal Points and Experts Group. Survey on the Use of Whole-Genome Sequencing for Infectious Diseases Surveillance: Rapid Expansion of European National Capacities, 2015-2016. Front Public Health. 2017;5:347. https://doi.org/10.3389/fpubh.2017.00347 PMID: 29326921 
License, supplementary material and copyright

This is an open-access article distributed under the terms of the Creative Commons Attribution (CC BY 4.0) Licence. You may share and adapt the material, but must give appropriate credit to the source, provide a link to the licence and indicate if changes were made.

Any supplementary material referenced in the article can be found in the online version.

This article is copyright of the authors or their affiliated institutions, 2020. 